\title{
On Switched Control of Networked System with Limited Communication and Handoff Delay
}

\author{
Zhiwen Wang ${ }^{1, a}$, Yewei $\mathrm{Yu}^{2, \mathrm{~b}}$, Li Xuan ${ }^{3, \mathrm{c}}$ \\ ${ }^{1}$ College of Electrical and Information Engineering, Lanzhou University of Technology, Lanzhou, \\ 730050, China \\ ${ }^{2}$ College of Electrical and Information Engineering, Lanzhou University of Technology, Lanzhou, \\ 730050, China \\ ${ }^{3}$ College of Electrical and Information Engineering, Lanzhou University of Technology, Lanzhou, \\ 730050, China
}

aemail:wzw@lut.cn, bemail: ccyuyewei@163.com, email: Illlixuan@163.com

Keywords: Average dwell time; Communications limited; Handoff delay

\begin{abstract}
In this paper, the issues of stability analysis and controller design of networked switched control system are discussed by considering the communication limitation and handoff delay simultaneously. Based on Lyapunov stability theory and linear matrix inequality, the minimum average dwell time and the controller design which can guarantee the system stability are presented. The feasibility of the proposed approach is verified by numerical simulation.
\end{abstract}

\section{Introduction}

Compared in the study of networked control systems, people have a longer time to study of hybrid system especially switched system. 'Switch' as a kind of control thoughts was applied to the control system very early. Now people generally accepted that switched system is a kind of hybrid systems with hierarchy type characteristics [1-3]. As a kind of important industrial system, switched system is widely used in the actual production. Relevant examples were visible in the literature [4][5]. In order to simplify the model, the research of networked switched control systems usually ignores handoff delay and only considers the transmission delay caused by the network, but due to the control system scale is more and more complex and precision require higher in actual industrial production, handoff delay caused by the subsystem switch will have to become one of the factors we consider that ensure the stability of the system [6][7].

Although communications technology has rapidly developed in recent years, but due to the amount of data transmission increased limited communications has become an important problem in network system. Limited communication restrictions feedback signal and control signal real-time transmission and reduce the performance even cause system instability, therefore need find an appropriate method to solve the problems of networked control system modeling, analysis and design with limited communication [8-15]. In this paper, on the basis of previous scholars' research and according to practical engineering, research design of switch controller for networked switched control system with limited communication and handoff delay, it has important significance for the actual industrial production.

\section{Modeling of Switched Control System with Limited Communication}

The switched system can be described as follow:

$$
\begin{aligned}
& \dot{x}(t)=A_{\Delta} x(t)+B_{\Delta} u(t) \\
& y(t)=C_{\Delta} x(t)
\end{aligned}
$$

Where $x=\left[x_{1}, x_{2}, \ldots x_{\mathrm{n}}\right]^{T} \in R^{n}, u=\left[u_{1}, u_{2}, \ldots u_{\mathrm{m}}\right]^{T} \in R^{m}, y=\left[y_{1}, y_{2}, \ldots y_{\mathrm{p}}\right]^{T} \in R^{p}$ are state, input and output of the system, respectively. $A_{\Delta} 、 B_{\Delta}$ and $C_{\Delta}$ are matrixes with appropriate dimensions. $\Delta$ is 
switch signal, satisfy set $\eta=\{1, \ldots N\}$. Assuming that system all $n$ state can be collected, but only $\sigma(1 \leq \sigma<p)$ sensor nodes and $\rho(1 \leq \rho<m)$ actuator node can communicate with the controller node every moment because of limited communication.

Introduce two valued function $\sigma_{i}(t): \mathrm{R} \rightarrow\{0,1\}$. Represents communication between $i(1 \leq i \leq p)$ sensor nodes and controller at time $t, \sigma_{i}(t)=0$ means not communication $\sigma_{i}(t)=1$ means communication. Communication between sensor nodes and controller can be expressed by the sequence $\sigma(t)=\left[\sigma_{1}(t) \sigma_{2}(t) \ldots \sigma_{p}(t)\right]^{T}$. Similarly, introduce two valued function $\rho_{j}(t): \mathrm{R} \rightarrow\{0,1\}$. Represents communication between $j(1 \leq j \leq m)$ actuator nodes and controller at time t. $\rho_{j}(t)=0$ means not communication $\rho_{j}(t)=1$ means communication. Communication between actuator nodes and the controller can be expressed by the sequence $\rho(t)=\left[\rho_{1}(t) \rho_{2}(t) \ldots \rho_{m}(t)\right]^{T}$. Controller receives output of the sensor node $i$ and the actuator node $j$ receives output of controller can expressed as $\bar{y}_{i}(t)=\sigma_{i}(t) y_{i}(t), \bar{u}_{j}(t)=\rho_{j}(t) u_{j}(t)$ respectively. In order to establish the communication model of networked control system with communication limitation definition $\bar{y}(t)=\left[\bar{y}_{1}, \bar{y}_{2}, \ldots, \bar{y}_{p}\right]^{T} \in R^{P}, \quad \bar{u}(t)=\left[\bar{u}_{1}, \bar{u}_{2}, \ldots, \bar{u}_{m}\right]^{T} \in R^{m}$, $M_{\sigma}(t)=\left(\begin{array}{ccc}\sigma_{1}(t) & & \\ & \ddots & \\ & & \sigma_{p}(t)\end{array}\right), M_{\rho}(t)=\left(\begin{array}{ccc}\rho_{1}(t) & & \\ & \ddots & \\ & & \rho_{m}(t)\end{array}\right)$. Utilize matrix form of communication sequence the original system model can be rewritten the following form, where $\bar{B}_{\Delta}(t)=B_{\Delta} M_{\rho}(t), \bar{C}_{\Delta}(t)=M_{\sigma}(t) C_{\Delta}$.

$$
\begin{aligned}
& \dot{x}(t)=A_{\Delta} x(t)+\bar{B}_{\Delta}(t) \bar{u}(t) \\
& \bar{y}(t)=\bar{C}_{\Delta}(t) x(t)
\end{aligned}
$$

The proposed system diagram is shown in Fig.1.

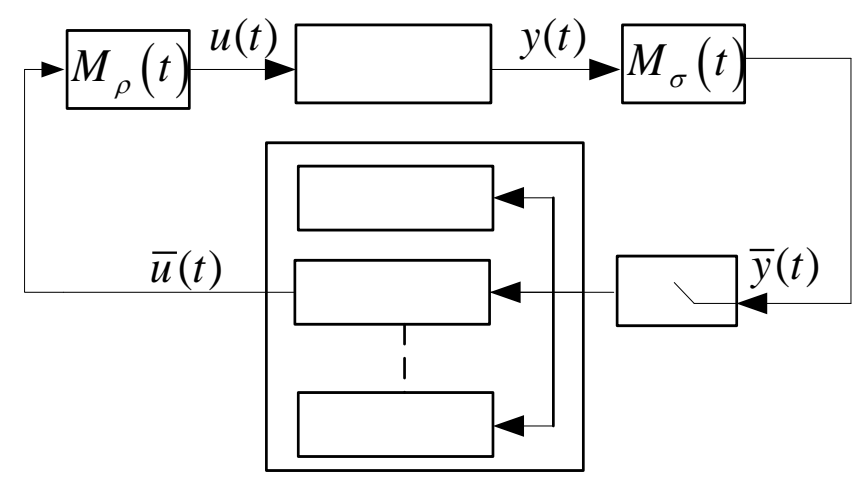

Fig. 1. Structure of networked switched control system with limited communication

\section{Controller Design of Limited Communication System with Handoff Delay}

Assumed that the maximum delay of switch is $\tau$ when the system switched between $N$ controllers, consider state feedback controller (3) exists in limited communication switched control system.

$$
\begin{aligned}
& \dot{x}_{c}(t)=A_{c, \Delta\left(t^{\prime}\right)} x_{c}(t)+B_{c, \Delta\left(t^{\prime}\right)} x(t) \\
& u(t)=C_{c, \Delta\left(t^{\prime}\right)} x_{c}(t)+D_{c, \Delta\left(t^{\prime}\right)} x(t)
\end{aligned}
$$

Where $t^{\prime}=t+\tau$, set up $X=\left[\begin{array}{ll}x & x_{c}\end{array}\right]^{T}$, then closed-loop networked switched control system with limited communication and handoff delay can be described as: 


$$
\begin{aligned}
& \dot{X}(t)=\left(\tilde{A}_{\Delta}+\tilde{B}_{\Delta} \tilde{K}_{\Delta\left(t^{\prime}\right)}\right) X(t) \\
& Y(t)=\tilde{C}_{\Delta}(t) X(t)
\end{aligned}
$$

Where $\tilde{A}_{\Delta}=\left(\begin{array}{cc}A_{\Delta} & 0 \\ 0 & 0\end{array}\right), \tilde{B}_{\Delta}=\left(\begin{array}{cc}0 & \bar{B}_{\Delta} \\ E & 0\end{array}\right)=\left(\begin{array}{cc}0 & B_{\Delta} M_{\rho}(t) \\ E & 0\end{array}\right), \tilde{C}_{\Delta}=\left[\begin{array}{ll}\bar{C}_{\Delta} & 0\end{array}\right]=\left[\begin{array}{ll}M_{\sigma}(t) C_{\Delta} & 0\end{array}\right], \tilde{K}_{\Delta\left(t^{\prime}\right)}=\left(\begin{array}{cc}B_{c, \Delta\left(t^{\prime}\right)} & A_{c, \Delta\left(t^{\prime}\right)} \\ D_{c, \Delta\left(t^{\prime}\right)} & C_{c, \Delta\left(t^{\prime}\right)}\end{array}\right)$.

Theorem: Considering system (4), for arbitrary $(i, j) \in \eta \times \eta$, $i \neq j$, let $\bar{Q}_{1, i}=R^{1 / 2} Q_{1, i} R^{1 / 2}$, $\bar{Q}_{2, i}=R^{1 / 2} Q_{2, i} R^{1 / 2}$ if there are matrixes $Q_{1, i}>0, Q_{2, i}>0, M_{i}$ and constant $b \geq a>0$ let the following condition were established:

$$
\begin{aligned}
& \tilde{A}_{i} Q_{i}+Q_{i} \tilde{A}_{i}^{T}+M_{i}^{T} \tilde{B}_{i}^{T}+\tilde{B}_{i} M_{i}-a Q_{i}<0 \\
& \tilde{A}_{i} Q_{j}+Q_{j} \tilde{A}_{i}^{T}+M_{j}^{T} \tilde{B}_{i}^{T}+\tilde{B}_{i} M_{j}-b Q_{j}<0 \\
& \frac{c_{1}}{\lambda_{1}}<\frac{c_{2}}{\lambda_{2}} e^{-\alpha T_{f}}
\end{aligned}
$$

Where $\mathrm{Q}_{i}=\left[\begin{array}{cc}Q_{1, i} & 0 \\ 0 & Q_{2, i}\end{array}\right]$ is positive definite matrix, switch signal $\Delta$ meet the average dwell time condition:

$$
\tau_{\alpha}>\tau_{\alpha}^{*}=\frac{T_{f}(\bar{\alpha}+\ln \mu)}{\ln \left(\frac{C_{2}}{\lambda_{2}}\right)-\ln \left(\frac{C_{1}}{\lambda_{1}}\right)-a T_{f}}
$$

For system (4), there are a series of state feedback controllers that make the closed-loop system about $\left(c_{1}, c_{2}, T_{f}, R, \Delta\right)$ finite time stable. Where $\mu=\lambda_{2} / \lambda_{1}, \quad \lambda_{1}=\min _{i \in \eta}\left(\lambda_{\text {min }}\left(\bar{Q}_{i}\right)\right)$, $\lambda_{2}=\max _{i \in \eta}\left(\lambda_{\max }\left(\bar{Q}_{i}\right)\right), \quad c_{1} \geq 1, \quad \bar{\alpha}=(b-a) \tau$ and controller gain $\tilde{K}_{i}=M_{i} Q_{i}^{-1}$.

\section{Prove:}

The stability analysis of complex switch system is usually based on Lyapunov stability theory, which mainly includes single Lyapunov function and multiple Lyapunov function. Multi Lyapunov function method is that each subsystem has its own Lyapunov function analysis method, which is more general than single Lyapunov function method [13][14].

Select multiple Lyapunov function $V_{i}(X)=X^{T} Q_{i}^{-1} X=x^{T} Q_{1, i}^{-1} X+x_{c}^{T} Q_{2, i}^{-1} x_{c}$, controller gain in the time of $\left[t_{k}, t_{k}+\tau\right]$ is $\tilde{K}_{j}$, in $\left[t_{k}+\tau, t_{k+1}\right]$ is $\tilde{K}_{i}$. In $\left[t_{k}, t_{k}+\tau\right]: \quad \dot{V}_{i}(t)-b V_{i}(t)=X^{T}(t)\left(\tilde{A}_{i}^{T} Q_{i}^{-1}+\tilde{K}_{j}^{T} \tilde{B}_{i}^{T} Q_{i}^{-1}+Q_{i}^{-1} \tilde{A}_{i}+Q_{i}^{-1} \tilde{B}_{i} \tilde{K}_{j}-b Q_{i}^{-1}\right) X(t)$, in $\left[t_{k}+\tau, t_{k+1}\right]: \quad \dot{V}_{i}(t)-a V_{i}(t)=X^{T}(t)\left(\tilde{A}_{i}^{T} Q_{i}^{-1}+\tilde{K}_{i}^{T} \tilde{B}_{i}^{T} Q_{i}^{-1}+Q_{i}^{-1} \tilde{A}_{i}+Q_{i}^{-1} \tilde{B}_{i} \tilde{K}_{i}-a Q_{i}^{-1}\right) X(t)$. Consider transformation as follow:

$$
\begin{aligned}
& Q_{i}^{-1}\left(\tilde{A}_{i} Q_{i}+Q_{i} \tilde{A}_{i}^{T}+M_{i}^{T} \tilde{B}_{i}^{T}+\tilde{B}_{i} M_{i}-a Q_{i}\right) Q_{i}^{-1} \\
& =Q_{i}^{-1} \tilde{A}_{i}+\tilde{A}_{i}^{T} Q_{i}^{-1}+Q_{i}^{-1} M_{i}^{T} \tilde{B}_{i}^{T} Q_{i}^{-1}+Q_{i}^{-1} \tilde{B}_{i}^{T} M_{i} Q_{i}^{-1}-Q_{i}^{-1} a \\
& =Q_{i}^{-1} \tilde{A}_{i}+\tilde{A}_{i}^{T} Q_{i}^{-1}+Q_{i}^{-1} \tilde{B}_{i}^{T} \tilde{K}_{i}+\tilde{K}_{i}^{T} \tilde{B}_{i}^{T} Q_{i}^{-1}-a Q_{i}^{-1}<0
\end{aligned}
$$

we can get $\dot{V}_{i}(t)-a V_{i}(t)<0 \quad t \in\left[t_{k}+\tau, t_{k+1}\right]$. Similarly $\dot{V}_{i}(t)-b V_{i}(t)<0 \quad t \in\left[t_{k}, t_{k}+\tau\right]$, it means that $V_{i}(t)$ drop along the trajectory for each subsystem, taking into account $b \geq a>0$, can get satisfy the entire time period $\left(t \in\left[t_{k}, t_{k+1}\right]\right): V_{\Delta(t)}\left(X_{t}\right) \leq e^{a\left(t-t_{k}\right)} e^{(b-a) \tau} V_{\Delta\left(t_{k}\right)}\left(X_{t_{k}}\right)$.

Based on the definition of $\lambda_{1}$ and $\lambda_{2}$ given before, can be known for any $i, j \in \eta, i \neq j$ the following inequality were established:

$$
X^{T}(t) \bar{Q}_{i}^{-1} X(t) \leq X^{T}(t) \lambda_{\max }\left(\bar{Q}_{i}^{-1}\right) X(t)=\frac{1}{\lambda_{\min }\left(\bar{Q}_{i}\right)} X^{T}(t) X(t) \leq \frac{1}{\lambda_{1}}\left(X^{T}(t) X(t)\right)
$$




$$
X^{T}(t) \bar{Q}_{j}^{-1} X(t) \geq X^{T}(t) \lambda_{\min }\left(\bar{Q}_{j}^{-1}\right) X(t)=\frac{1}{\lambda_{\max }\left(\bar{Q}_{j}\right)} X^{T}(t) X(t) \geq \frac{1}{\lambda_{2}}\left(X^{T}(t) X(t)\right)
$$

Then can get: $X^{T}(t) \bar{R}^{1 / 2} Q_{i}^{-1} \bar{R}^{1 / 2} X(t) \leq \mu X^{T}(t) \bar{R}^{1 / 2} Q_{j}^{-1} \bar{R}^{1 / 2} X(t)$ where $\bar{R}^{1 / 2}=\left(\begin{array}{cc}R^{1 / 2} & 0 \\ 0 & R^{1 / 2}\end{array}\right)$. It can be rewritten $V_{i}\left(X_{t_{k}}(t)\right) \leq \mu V_{j}\left(X_{t_{k}}(t)\right)$, because the system status does not jumping for any time $t \in\left(0, T_{f}\right)$, then $V_{\Delta(t)}\left(X_{t_{k}}(t)\right) \leq \mu V_{\Delta\left(t_{k}\right)}\left(X_{t_{k}}(t)\right)$. In consideration of $\mu \geq 1$ and $N_{\Delta}(0, t) \leq N_{0}+t / \tau_{\alpha} \leq T_{f} / \tau_{\alpha}$ can get:

$$
\begin{aligned}
V_{\Delta(t)}\left(X_{t}(t)\right) & \leq e^{a\left(t-t_{k}\right)} e^{(b-a) \tau} V_{\Delta\left(t_{k}\right)}\left(X_{t_{k}}\right) \\
& \leq \mu e^{a\left(t-t_{k}\right)} e^{(b-a) \tau} V_{\Delta\left(t_{k}^{-}\right)}\left(X_{t_{k}}\right) \\
& =\mu e^{a\left(t-t_{k}\right)} e^{(b-a) \tau} V_{\Delta\left(t_{k-1}\right)}\left(X_{t_{k}}\right) \\
& <\mu e^{a\left(t-t_{k}\right)} e^{(b-a) \tau} e^{a\left(t_{k}-t_{k-1}\right)} e^{(b-a) \tau} \mu V_{\Delta\left(t_{k-1}\right)}\left(X_{t_{k-1}}\right) \\
& \vdots \\
& <\left[\mu e^{\bar{\alpha}}\right]^{N_{\Delta}(0, t)} e^{a\left(t-t_{k}\right)} e^{a\left(t_{k}-t_{k-1}\right)} \cdots e^{a\left(t_{1}-t_{0}\right)} V_{\Delta(0)}\left(X_{0}(t)\right) \\
& =e^{a t}\left[\mu e^{\bar{\alpha}}\right]^{N_{\Delta}(0, t)} V_{\Delta(0)}\left(X_{0}(t)\right) \\
& <e^{a t}\left[\mu e^{\bar{\alpha}}\right]^{T_{f} / \tau_{\alpha}} V_{\Delta(0)}\left(X_{0}(t)\right) \\
& \leq e^{(\bar{\alpha}+\ln \mu)\left(T_{f} / \tau_{\alpha}\right)}\left[e^{a T_{f}} V_{\Delta(0)} X_{0}(t)\right]
\end{aligned}
$$

On account of:

$$
\begin{aligned}
\mathrm{V}_{\Delta(t)}(X(t))= & x^{T}(t) Q_{1, \Delta(\mathrm{t})}^{-1} x(t)+x_{c}^{T}(t) Q_{2, \Delta(\mathrm{t})}^{-1} x_{c}(t) \geq \lambda_{\min }\left(\bar{Q}_{1, \Delta(t)}^{-1}\right) x^{T}(t) R x(t) \geq \frac{1}{\lambda_{2}} x^{T}(t) R x(t) \\
\mathrm{V}_{\Delta(0)}\left(X_{0}(0)\right) & =x^{T}(0) Q_{1, \Delta(0)}^{-1} x(0)+x_{c}^{T}(0) Q_{2, \Delta(0)}^{-1} x_{c}(0) \\
& =x^{T}(0) Q_{1, \Delta(0)}^{-1} x(0) \\
& \leq \lambda_{\max }\left(\bar{Q}_{1, \Delta(0)}^{-1}\right) x^{T}(0) R x(0) \\
& \leq \frac{1}{\lambda_{1}} x^{T}(0) R x(0)
\end{aligned}
$$

therefore $x^{T}(t) R x(t) \leq \lambda_{2} V_{\Delta(t)}(X(t))<\frac{\lambda_{2} C_{1}}{\lambda_{1}} e^{(\bar{\alpha}+\ln \mu)\left(T_{f} / \tau_{\alpha}\right)+a T_{f}}$, when the conditions $\frac{C_{1}}{\lambda_{1}}<\frac{C_{2}}{\lambda_{2}} e^{-a T_{f}}$ and $\ln \left(\frac{c_{2}}{\lambda_{2}}\right)-\ln \left(\frac{c_{1}}{\lambda_{1}}\right)-a T_{f}>0$ are met, can be obtained $\frac{T_{f}}{\tau_{\alpha}} \leq \frac{\ln \left(\frac{c_{2}}{\lambda_{2}}\right)-\ln \left(\frac{c_{1}}{\lambda_{1}}\right)-a T_{f}}{\bar{\alpha}+\ln \mu}$ by average dwell time formula, then $e^{(\bar{\alpha}+\ln \mu)\left(T_{f} / \tau_{\alpha}\right)} \leq \frac{\lambda_{1} C_{2}}{\lambda_{2} c_{1} e^{a T_{f}}} \quad$ was derived. Substitutes the formula to $x^{T}(t) R x(t) \leq \lambda_{2} V_{\Delta(t)}(X(t))<\frac{\lambda_{2} C_{1}}{\lambda_{1}} e^{(\bar{\alpha}+\ln \mu)\left(T_{f} / \tau_{\alpha}\right)+a T_{f}}$ then $x^{T}(t) R x(t)<c_{2}$.

If there are three positive real numbers $c_{1}, c_{2}\left(c_{1}<c_{2}\right), T_{f}$, a positive definite matrix $R$ and switch signal $\Delta$ in a networked control system, when the input and disturbance of the networked switched control system are zero and the system satisfies the following inequality: $x_{0}^{\mathrm{T}}(t) R x_{0}(t)<c_{1} \Rightarrow x^{\mathrm{T}}(t) R x(t)<c_{2}, \quad \forall t \in\left[0, T_{f}\right]$ the networked switched control system is stability about $\left(c_{1}, c_{2}, \mathrm{~T}_{f}, R, \Delta\right)$. In summary, closed-loop networked switched control system (4) is finite time stability. 


\section{Simulation Verification and Analysis}

Consider networked control system (2) with limited communication and handoff delays, system switch before and after matrix are as follows:

$$
A_{1}=\left[\begin{array}{cc}
-2 & 1.2 \\
-0.3 & -1.5
\end{array}\right] \bar{B}_{1}=\left[\begin{array}{ll}
0 & 2 \\
1 & 0
\end{array}\right], A_{2}=\left[\begin{array}{cc}
-1 & 0.9 \\
0.5 & -3
\end{array}\right] \bar{B}_{2}=\left[\begin{array}{cc}
0.5 & -0.2 \\
1.3 & 2
\end{array}\right]
$$

Select parameters $a=0.02, b=1.1, R=I, c_{1}=1, c_{2}=25, T_{f}=5$ handoff delay $\tau=0.05 \mathrm{~s}$, by the linear matrix inequalities in the theorem, using the LMI toolbox in MATLAB can get the following matrix to prove the method is feasible:

$$
\begin{aligned}
Q_{1} & =\left[\begin{array}{cc}
38.5304 & 5.5027 \\
5.5027 & 34.1334
\end{array}\right], Q_{2}=\left[\begin{array}{cc}
30.8979 & 8.7130 \\
8.7130 & 39.8057
\end{array}\right] \\
M_{1} & =\left[\begin{array}{cc}
1.1077 & 25.0513 \\
30.2814 & -6.9043
\end{array}\right], M_{2}=\left[\begin{array}{cc}
14.7620 & -39.3663 \\
4.1789 & 71.9879
\end{array}\right]
\end{aligned}
$$

For any switch signal $\Delta$, get average dwell time $\tau_{a}>\tau_{a}^{*}=1.1951 \mathrm{~s}$. It means when the average dwell time of the switch signal is larger than the minimum average dwell time 1.1951s system is finite time stable about $(1,25,5, I, \Delta)$, we obtained the controller parameter matrix as following: $\tilde{K}_{1}=\left(\begin{array}{cc}-0.0783 & 0.7468 \\ 0.8352 & -0.3373\end{array}\right), \tilde{K}_{2}=\left(\begin{array}{cc}0.8085 & -1.1751 \\ -0.4029 & 1.9119\end{array}\right)$. The simulation results are shown in figure, figure 2 shows for a defined constant $c_{1}$, effects of $c_{2}$ changes on average dwell time, figure 3 shows the response curve of close-loop state.

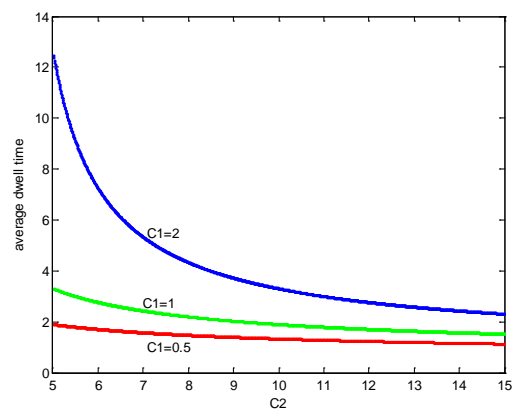

Fig. 2. Average dwell time $\tau_{a}^{*}$ at different $c_{2}$

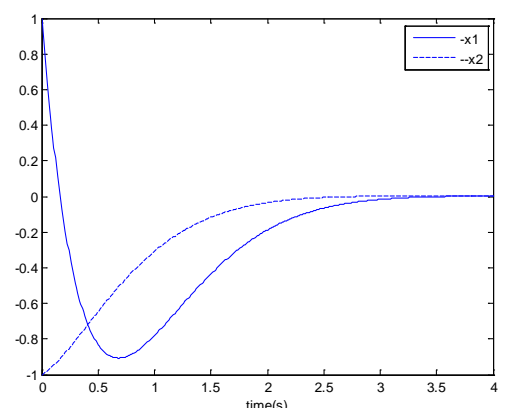

Fig. 3. System close-loop state response curve

In Fig. 2 the minimum value of the average dwell time decreases with the increase of $c_{2}$ when $c_{1}$ value is fixed. Fig. 3 is system closed-loop state response curve when the initial value $x=\left[\begin{array}{ll}1 & -1\end{array}\right]^{T}$, from the curve it can be seen that the state response of the system is stable, it is shown that the controller design method mentioned in this paper is effective in the presence of handoff delay and limited communication.

\section{Conclusion}

In this paper, we addressed the controller design problem for networked switched control system with limited communication and handoff delay using Lyapunov theory and LMI methods. Firstly, the model of system is analyzed. On the basis of analyzed, the design method of the controller is given by using the concept of average dwell time. Finally, the feasible solution is obtained by the linear matrix inequality method, which verifies the validity and feasibility of the design method. Future work aims to address performance issues such as how to obtain more general stability criteria and how to choose the controller design parameters to improve transient response. Also, the influence of network transmission performance on the networked control system. 


\section{Acknowledgement}

In this paper, the research was sponsored by the Natural Science Foundation of China under grants (Project No. 61263003) and Natural Science Foundation of China (Project No. 61563031).

\section{References}

[1] Lin H, Antsaklis P J. Stability and stabilizability of switched linear systems: A survey of recent result [J]. IEEE Transactions on Automatic Control, 2009, 54(2):308-322.

[2] Li J N, Zhang Q L, Cai M. Modelling and robust stability of networked control systems with packet reordering and long delay [J]. Int. J. Contr., 2009, 82(10): 1773-1785.

[3] Donkers M C F, Heemels W P M H, van de Wouw N, et al. Stability analysis of network control systems using a switched linear systems approach [J]. IEEE Transactions on Automatic Control, 2011, 56(9): 2101-2115.

[4] Liberzon D. Switching in Systems and Control [M]. Boston, Birkhuaser, 2003.

[5] Sun Z D, GE S S. Switched Linear Systems-Control and Design [M]. New York: Springer-Verlag, 2004.

[6] Kim D K, Park P G., Ko J W. Output-feedback ${ }^{\infty}{ }$ control of systems over communication networks using a deterministic switching system approach [J]. Automatic, 2004, 40(7), 1205-1212.

[7] Blanchini F, Colaneri P, Valcher M.E. Co-Positive Lyapunov Functions for the Stabilization of Positive Switched Systems [J]. IEEE Transactions, 2012, 57(12): 3038-3050.

[8] Gao H J, Chen T W. A new delay system approach to network-based control [J]. Automatic, 2008, 44(1): 39-52.

[9] Martins E C, Jota F G. Design of networked control systems with explicit compensation for time-delay variations [J]. IEEE Trans Syst Man Cy C, 2010, 40(3): 308-318.

[10] Lu Z B, Guo G. Communications and control co-design: a combined dynamic-static scheduling approach [J]. Science China Information Sciences, 2012, 55(55):2495-2507.

[11] Guo G, Jin H. A switching system approach to actuator assignment with limited channels [J]. Int J Robust Nonlin, 2009, 20(12): 1407-1426.

[12] Guo G. A switching system approach to sensor and actuator assignment for stabilization via limited multi-packet transmitting channels [J]. Int J Control, 2011, 84(1): 78-93.

[13] Wang D, Wang W and Shi P. Exponential ${ }_{\infty}$ Filtering for Switched Linear Systems with Interval Time-varying Delay [J]. Robust And Nonlin Control, 2009, 41(5): 532-551.

[14] Zhang L and Gao H. Asynchronously Switched Control of Switched Linear Systems with Average Dwell time [J]. Automatic, 2010, 46(5): 953-958. 